Izquierdo Rus, T., Asensio Martínez, E., Escarbajal Frutos, A. y Rodríguez Moreno, J. (2019). El aprendizaje cooperativo en la formación de maestros de Educación Primaria. Revista de Investigación Educativa, 37(2), 543-559. DOI: http://dx.doi.org/10.6018/rie.37.2.369731

\title{
El aprendizaje cooperativo en la formación de maestros de Educación Primaria
}

\section{Cooperative learning in teachers' teaching of Primary Education}

\author{
Tomás Izquierdo Rus*, Elena Asensio Martínez*, Andrés Escarbajal Frutos ${ }^{* * *}$ y Javier Rodríguez \\ Moreno***** \\ *Departamento de Métodos de Investigación y Diagnóstico en Educación. Facultad de Educación. Universidad de \\ Murcia (España) \\ ** Departamento de Métodos de Investigación y Diagnóstico en Educación. Facultad de Educación. Universidad de \\ Murcia (España) \\ ***Departamento de Didáctica y Organización Escolar. Facultad de Educación. Universidad de Murcia (España) \\ ****Departamento de Pedagogía. Facultad de Humanidades y Ciencias de la Educación. Universidad de Jaén (España)
}

\section{Resumen}

La universidad es un contexto de aprendizaje, en el que el alumnado es el responsable de tomar sus propias decisiones, controlar su propio proceso de aprendizaje y las interacciones que intervienen en él. En los grados como el de Educación Primaria, el progreso del aprendizaje no depende sólo de un individuo, sino del esfuerzo conjunto de un grupo de iguales, cuyo desempeño se ve reflejado en el aprendizaje individual. Así, los objetivos del presente trabajo han sido establecer cómo se desarrolla el concepto de trabajo en grupo y las estrategias que ponen en marcha para llevarlo a cabo en función de su satisfacción. Los participantes de esta investigación han sido 523 estudiantes del Grado de Educación Primaria de las Universidades de Murcia y de Jaén. Para ello se ha utilizado un cuestionario tipo Likert con cuyos resultados se ha establecido una comparación entre el alumnado que inicia el grado en Educación Primaria y aquellos que ya tienen varios años de experiencia en el ambiente universitario. Destacar como principales resultados y conclusiones que el alumnado desarrolla un concepto del trabajo en grupo que se mantiene estable en el tiempo. Sin embargo, la utilidad que creen que les reporta el trabajo en grupo sí puede cambiar, influenciados por las exigencias del contexto. Así mismo, cuando el

Correspondencia: Javier Rodríguez Moreno, jrmoreno@ujaen.es, Departamento de Pedagogía, Facultad de Humanidades y Ciencias de la Educación, Universidad de Jaén, 23071 Jaén (España). 
alumnado está satisfecho con el trabajo de su grupo confían más en sus propias habilidades y capacidades para construir su conocimiento.

Palabras clave: educación superior; estudiante universitario; enseñanza profesional; aprendizaje cooperativo.

\section{Abstract}

The university is a learning context, where the students are responsible for making their own decisions and controlling their own learning process and the interactions that intervene in it. Not only that, in degrees such as Primary Education, the progress of learning does not depend only on an individual, but on the joint effort of a peer group, whose performance is reflected in the individual learning. Thus, the objectives of this paper have been to establish how the concept of group work is developed and the strategies that are put in place to carry it out according to their satisfaction. The participants of this research have been 523 students of the Degree of Primary Education of the University of Murcia and the University of Jaen. To this end, a Likert questionnaire has been used, with the results of which a comparison has been established between the students who start the degree in Primary Education and those who already have several years of experience in the university environment. The main results and conclusions confirm that the students develop a concept of group work that remains stable over time. However, the usefulness that they believe that group work brings can change, influenced by the demands of the context. Additionally, when students are satisfied with the work of their group, they rely more on their own skills and abilities to build their knowledge.

Keywords: higher education; University students; vocational education; cooperative learning.

\section{Introducción}

En la actualidad, desde el nuevo marco de desarrollo de competencias y con el propósito de ajustar el aprendizaje a un contexto más real y que reconozca los aspectos sociales del aprendizaje, las instituciones educativas, en todos los niveles de la educación, promueven el trabajo junto con otras personas en lugar del individual.

El trabajo en grupo se caracteriza por la negociación que establecen todos los participantes durante las distintas fases de la tarea con el fin de consensuar una respuesta común que todos conocen, aceptan y de la que se hacen responsables por igual. Se establece, un canal de comunicación fluida entre los miembros del grupo, como apunta Domingo (2008), vínculos de colaboración, solidaridad y desarrollo de las características personales.

Antúnez (1999) define el trabajo colaborativo como el trabajo en grupo que se desarrolla de manera voluntaria y entre iguales, de manera que no existe autoridad jerárquica alguna. Esto viene a significar que, aunque los miembros del grupo comparten el objetivo de alcanzar un mismo fin, este propósito no le ha sido impuesto por lo que lleva asociadas connotaciones democráticas de igualdad a lo largo del proceso educativo. Por otra parte, el trabajo cooperativo se entiende como una estructura de trabajo en la que las decisiones del alumnado están supeditadas a las directrices y condiciones establecidas por el docente (Laal \& Laal, 2012; Panitz, 1999). 


\section{El aprendizaje cooperativo en Educación Superior}

La organización de las actividades académicas en grupos de trabajo reducidos conlleva una reestructuración de la configuración de la enseñanza y el aprendizaje en la que cambian las relaciones alumno-profesor y alumno-alumno. Esta metodología es el punto de inflexión que cambia el foco de la clase de la enseñanza del docente al aprendizaje del alumnado.

Sin embargo, hay que tener en cuenta que estos beneficios no se dan de manera automática al agrupar al alumnado (Escarbajal, 2010), sino que requieren un esfuerzo de adaptación por parte de los alumnos al nuevo planteamiento de trabajo, así como la planificación de las dinámicas y objetivos adecuados por parte del profesor. Por esa razón, estas propuestas pueden generar desconcierto si no se emplean de manera adecuada y ajustada a las necesidades y propósitos de la actividad (Galán \& Fuentes, 1999).

Serrano (1996) menciona algunos de los beneficios que posee el aprendizaje cooperativo y su potencial frente al individual, como son:

El incremento del rendimiento de los alumnos independientemente de su edad o del número de actividades a realizar, puesto que el compromiso con el resto del equipo lleva a la mayor implicación en la tarea.

Aumenta la motivación intrínseca hacia el aprendizaje.

Produce actitudes positivas tanto hacia el aprendizaje como hacia el profesor y los compañeros.

Tiene una fuerte correlación positiva con niveles superiores de autoestima.

Crea en el estudiante la percepción de que los demás compañeros se interesan y desean ayudarle en su proceso de aprendizaje.

Favorece la aceptación de compañeros de otras etnias.

Facilita la inclusión de los alumnos con necesidades educativas especiales.

Además, se ha comprobado que las relaciones entre iguales favorecen la formación de competencias, así como los procesos y habilidades de socialización al tiempo que controla las respuestas agresivas. Este contexto social de aprendizaje también da lugar al incremento de las aspiraciones de los alumnos al compararlas y ponerlas en valor frente a las de los otros compañeros.

Y, según Johnson, Johnson y Holubec (1999), el aprendizaje cooperativo se caracteriza por estas cinco dimensiones:

- Interdependencia positiva: tiene lugar cuando cada uno de los miembros del grupo entiende que su éxito se mide en función del éxito que consiguen los demás integrantes del grupo y, con ello, el grupo en sí.

- Responsabilidad individual y de equipo: tiene lugar cuando todos los componentes del grupo asumen la responsabilidad de hacerse cargo de la tarea que les es encomendada y a la vez conocer, y si es necesario responder, por la tarea de cualquier otro.

- Interacción estimuladora: es la realizada cara a cara entre los miembros del equipo y durante la que se apoyan en su proceso de aprendizaje compartiendo sus recursos, conocimientos y experiencias pasadas, y conectando sus propios 
conocimientos mientras que se apoyan, animan y felicitan a los demás por el trabajo realizado.

- Habilidades sociales y de trabajo en grupo: son habilidades que los estudiantes aprenden a utilizar como resultado de la necesidad de interacción. Incluyen mecanismos de negociación, toma de decisiones y resolución de conflictos; creación de vías comunicativas, propuesta de ideas creativas y críticas constructivas y también su aceptación.

- Reflexión del grupo: con ella se evalúa el funcionamiento y la dinámica del grupo, así como su rendimiento y la medida en la que sus resultados se ajustan a los objetivos propuestos. A partir de ella se pueden establecer cambios y propuestas de mejora para futuros trabajos, asegurándose de que todos los miembros reciben feedback acerca de los aspectos positivos y los puntos mejorables de su intervención.

Por lo tanto, se ratifica que el trabajo en equipo (Stortoni, 2016), es una capacidad particularmente importante dentro del ejercicio docente ya que múltiples estudios avalan con claridad que ésta repercute de manera determinante en la calidad de la educación que se consigue en los centros educativos. El trabajo en equipo entre docentes les ayuda a la planificación y a la ejecución estructurada y a crear cohesión, colaboración y consenso (Conde, Martín, \& Torres, 2012; Krichesky \& Murillo, 2018).

\section{La competencia del trabajo en equipo y su importancia con el ámbito profesional del maestro}

En el contexto de la enseñanza universitaria, la valoración y consideración de la importancia del trabajo en equipo para el desarrollo de competencias profesionales ha producido un incremento importante de experiencias que lo utilizan como núcleo fundamental de sus propuestas.

No obstante, existen diferencias entre el concepto de competencia en el ámbito educativo y en el laboral (Izquierdo, Escarbajal \& Latorre, 2016). El Instituto Nacional para el Empleo define la competencia profesional como aquella que permiten el desempeño de una ocupación a los niveles requeridos. Además, va más allá del conocimiento técnico, al comportar saber y saber hacer, así como un conjunto de comportamiento y facultades que permiten la toma de decisiones, el análisis y la transmisión de información (Instituto Nacional para el Empleo [INEM], 1995).

De igual forma, la Agencia Nacional de Evaluación de la Calidad y Acreditación (ANECA, 2004), en su libro blanco refiere competencias del docente como una competencia de carácter personal y de saber estar necesaria para trabajar en un contexto social. Perrenoud (2004) también lo incluye dentro de sus diez competencias para enseñar como una competencia de referencia prioritaria para el profesorado, en concreto el de Primaria, y afirma que todo maestro debería ser capaz de coordinar actividades grupales desde organizar un pequeño grupo de trabajo hasta elaborar un proyecto de equipo.

Por lo tanto, se ratifica que el trabajo en equipo es una capacidad particularmente importante dentro del ejercicio docente ya que múltiples estudios avalan con clari- 
dad que esta repercute de manera determinante en la calidad de la educación que se consigue en los centros educativos. El trabajo en equipo entre docentes ayuda a la planificación y a la ejecución estructurada y a crear cohesión, colaboración y consenso entre el profesorado (Conde et al., 2012).

El trabajo en equipo es un valor añadido dentro de cualquier institución y, en concreto dentro del centro escolar, permite una acción sinérgica que siempre será más eficaz y efectiva que la individual, contribuye al análisis común de necesidades y problemas, así como a proporcionar una educación de calidad basada en principios comunes y compartidos; $y$, no menos importante, ayuda a que los maestros desarrollen un sentimiento de pertenencia que los una al centro escolar (Conde et al., 2012).

De esta manera se incentiva una cultura organizacional que según autores como Vázquez (2013) o Iglesias, González y Fernández-Río (2017) denominan de tipo clan y en la que sus miembros comparten metas y valores de manera cohesionada y participativa, acrecentando así un sentimiento de igualdad y valoración de las opiniones que rebasa la mera estructura del centro y repercute directamente en la educación integral de los alumnos (Murillo, Krichesky, Castro \& Reyes, 2010).

Sin embargo, un estudio de Merino, Cortón y Cortón (2012) con alumnos de magisterio manifiesta que el $76 \%$ de estos tienen la percepción de que se incentiva muy poco dentro de su titulación la competencia de trabajar en equipo con otros docentes, mientras que el $44 \%$ de sus profesores consideran que los alumnos han adquirido esta competencia bastante o mucho, coincidiendo con estudios como los de Gómez y Gómez (2016).

Las personas capaces de trabajar colaborativamente no sólo tienen una buena actitud hacia sus semejantes, sino que entienden que aprender es algo que se hace todos los días y que la manera de conseguirlo es a través de otras personas, y que es necesario trabajar todos los días codo con codo con los compañeros para seguir creciendo y enriqueciendo la propia vida y las de los demás en todas las competencias (Colomer, Durán \& Puigcerver, 2016; Rondón, Salas, González, Martínez \& González, 2018).

\section{Método}

El diseño del presente estudio es de corte cuantitativo no experimental ya que pretende dar a conocer la relación entre ciertas variables, donde ninguna de ellas se manipula intencionadamente; sólo se observan los fenómenos presentes en un lugar y tiempo determinados.

Según su secuencia es de corte transversal, pues la recogida de datos se establece en un solo momento en el tiempo. El alcance de esta investigación es descriptivo ya que, tal como las clasifican Hernández, Fernández y Baptista (2010), las investigaciones transversales descriptivas intentan medir las modalidades o niveles en los que se presentan una o varias variables dentro de una población.

Este estudio, además, es una investigación descriptiva de tipo comparativo, puesto que su objetivo es examinar las diferencias respecto a las variables seleccionadas dentro de tres grupos de población: los estudiantes de primer, segundo y tercer curso de la titulación de Grado en Educación Primaria de las Universidades de Murcia y de Jaén. 


\section{Objetivos}

Para este trabajo se han planteado dos objetivos, siguiendo del manual de Hernández (2006) las indicaciones para su formulación:

1. Conocer y comparar la opinión de los alumnos del Grado de Primaria sobre su concepto del trabajo en grupo, sobre lo que aporta a su formación y las estrategias que emplean para llevar a cabo el trabajo al inicio de su formación y una vez que han ganado experiencia con esta metodología.

2. Determinar la concepción que tiene el alumnado del trabajo en grupo y las estrategias que ponen en marcha para llevarlo a cabo en función de su satisfacción.

\section{Participantes}

Han participado en la investigación 523 estudiantes del Grado en Educación Primaria de dos universidades españolas: Universidad de Murcia y Universidad de Jaén. Del total, 322 se encuentran matriculados en la Universidad de Murcia (61.6\%) y 201 en la Universidad de Jaén (38.4\%). Por cursos, 250 estudiantes son de primer curso (47.8\%), 121 de segundo curso (23.1\%) y 152 de tercer curso (29.1\%). Según sexo, participan 142 hombres (27.2\%) y 381 mujeres (72.8\%) de entre 18 y 50 años, situándose la media de edad en 20.40 años. El muestreo probabilístico seguido en todos los cursos es el aleatorio simple.

De entre ellos, un total de 61 tienen empleo, siendo éstos 18 hombres y 43 mujeres. El rango de edad de las personas empleadas va de 18 a 45 años y su media de edad es de 23.7 años. El número de desempleados es de 462, 124 hombres y 381 mujeres, cuyo rango de edad va de 18 a 50 años, siendo la media de 19.9 años.

De cara a los intereses de esta investigación es conveniente mostrar la distribución de la población de acuerdo a otras variables, aparte de las demográficas. Esto se refiere a cómo se distribuye la muestra de acuerdo a la cantidad de trabajo que han llevado a cabo en grupo con las mismas personas, siendo la opción más elegida en la mayoría de las asignaturas, con un $94.5 \%$ y la satisfacción que sienten de acuerdo al resultado del trabajo del grupo un $90.1 \%$, con su propio aporte, un $95.8 \%$ y con la contribución de sus compañeros a la consecución de los objetivos propuestos, un $84.7 \%$.

\section{Instrumento}

El instrumento de recogida de información empleado en esta investigación es un cuestionario de preguntas cerradas basado en varias escalas tipo Likert. No obstante, puesto que estas escalas han sido extraídas de cuestionarios validados y empleados en otras investigaciones, se pasa a describirlos específicamente.

La primera parte del cuestionario está elaborada a partir del cuestionario de Análisis del trabajo cooperativo en Educación Superior de García, González y Mérida (2012). Concretamente, se han elegido 3 de sus dimensiones: concepción del trabajo en equipo (5 ítems), la utilidad del trabajo en grupo para su formación (6 ítems) y al funcionamiento 
interno de los grupos (7 ítems), expresadas en una escala tipo Likert de 1 a 5, donde 1 significa totalmente en desacuerdo y 5 totalmente de acuerdo. El alfa de Cronbach obtenido durante su validación fue de $\alpha=.844$, y el de las dimensiones mencionadas fue respectivamente $\alpha=.665, \alpha=.785$ y $\alpha=.666$.

La segunda parte del instrumento es la Voluntad de Trabajo en su versión para evaluar a los pares de Abal, Lozzia, Aguerri, Galibert y Attorresi (2008), consta de 16 ítems expresados en una escala tipo Likert de 1 a 7 , etiquetadas atendiendo a la frecuencia de la siguiente forma: nunca, casi nunca, pocas veces, a veces, con frecuencia, casi siempre y siempre. Tiene un rango de puntuaciones de 16 a 112 puntos y un alfa de Cronbach obtenido con una muestra procedente de Buenos Aires de $\alpha=.89$ y otro con muestra española de $\alpha=.92$ (Andrés et al., 2013).

\section{Procedimiento de recogida y análisis de datos}

Se contactó con los docentes de primer, segundo y tercer curso del Grado de Educación Primaria. En estas comunicaciones se les informó de la investigación y se acordó el día y la hora para pasar por sus clases. La actividad se iniciaba con una breve charla sobre la importancia de su colaboración. Así mismo, se les daba una serie de instrucciones acerca de cómo rellenar los cuestionarios.

Posteriormente, para el análisis de los datos se utilizó el programa de análisis estadístico SPSS (versión 19), empleándose estadísticos descriptivos y pruebas ANOVA para muestras independientes. No obstante, debido a que las muestras seleccionadas para algunas de estas pruebas pueden ser muy pequeñas, se procedió a la realización de la prueba Kolmogorov-Smirnov para verificar que todas las muestras analizadas con la prueba ANOVA seguían una distribución normal. En el caso de que una de ellas no lo ratificase, se procedía a realizar la prueba no paramétrica equivalente, es decir, la prueba Kruskal-Wallis para muestras independientes.

\section{Resultados y Discusión}

En relación al primer objetivo, tal y como puede observarse en la Tabla 1, los alumnos muestran estar muy de acuerdo con los primeros enunciados, puntuándolos dentro de un rango alto entre un 4.28 y 4.45 . Cabe señalar, sin embargo, que existen diferencias estadísticamente significativas en la opinión de los alumnos en lo referente a la capacidad del trabajo en grupo para desarrollar las competencias sociales $(p=.042)$; también, en la opinión de que el trabajo en grupo es compartir el volumen de trabajo $(\mathrm{p}=.043)$ y conocimientos $(\mathrm{p}=.040)$ y, por último, que trabajar en grupo ayuda a preparar los exámenes $(\mathrm{p}=.015)$.

Finalmente se muestran de acuerdo los tres grupos, con la idea de que el trabajo en grupo es una oportunidad para conocer mejor a sus compañeros, asignándoles a este aspecto una puntuación entre cuatro y cinco.

En lo que respecta a la utilidad que le conceden al trabajo en grupo, se aprecia que los resultados de los tres grupos, son similares; si bien los alumnos de segundo son marginalmente superiores. Como puede verse en la Tabla 2, los alumnos de primero están entre muy y absolutamente de acuerdo con todas las dinámicas. 
Tabla 1

Comparación de la concepción del trabajo en grupo en alumnos de primero, segundo y tercero

\begin{tabular}{|c|c|c|c|c|c|c|c|}
\hline \multirow[t]{2}{*}{ El trabajo en grupo es: } & \multicolumn{2}{|c|}{ Primero, $n=250$} & \multicolumn{2}{|c|}{$\begin{array}{l}\text { Segundo, } \mathrm{n}= \\
121\end{array}$} & \multicolumn{2}{|c|}{$\begin{array}{l}\text { Tercero, } \mathrm{n}= \\
152\end{array}$} & \multirow[t]{2}{*}{$\mathrm{p}$} \\
\hline & $\mathrm{M}$ & D.T. & $M$ & D.T. & $\mathrm{M}$ & D.T. & \\
\hline $\begin{array}{l}\text { Una oportunidad para conocer me- } \\
\text { jor a mis compañeros/as }\end{array}$ & 4.45 & .755 & 4.58 & 692 & 4.39 & .772 & .107 \\
\hline $\begin{array}{l}\text { Un buen método para desarrollar } \\
\text { mis competencias sociales: argu- } \\
\text { mentación, diálogo, capacidad de } \\
\text { escucha, debate, respeto a opinio- } \\
\text { nes discrepantes... }\end{array}$ & 4.28 & .814 & 4.50 & .743 & 4.35 & .783 & .042 \\
\hline $\begin{array}{l}\text { Una manera de compartir el volu- } \\
\text { men de trabajo total }\end{array}$ & 3.96 & 1.046 & 4.24 & 1.033 & 4.13 & 1.005 & .043 \\
\hline $\begin{array}{l}\text { Una forma de comprender mejor } \\
\text { los conocimientos }\end{array}$ & 3.90 & .938 & 4.17 & .960 & 3.99 & .980 & .040 \\
\hline $\begin{array}{l}\text { Una manera de facilitar la prepara- } \\
\text { ción de los exámenes }\end{array}$ & 3.50 & 1.10 & 3.72 & 1.112 & 3.30 & 1.234 & .015 \\
\hline
\end{tabular}

Tabla 2

Percepción de la utilidad del trabajo en grupo en alumnos de primero, segundo y tercero

\begin{tabular}{|c|c|c|c|c|c|c|c|}
\hline \multirow[t]{2}{*}{ El trabajo en grupo me ayuda a: } & \multicolumn{2}{|c|}{ Primero, $\mathrm{n}=250$} & \multicolumn{2}{|c|}{$\begin{array}{c}\text { Segundo, } \mathrm{n}= \\
121\end{array}$} & \multicolumn{2}{|c|}{$\begin{array}{l}\text { Tercero, } \mathrm{n}= \\
152\end{array}$} & \multirow[t]{2}{*}{$\mathrm{p}$} \\
\hline & $\mathrm{M}$ & D.T. & $\mathrm{M}$ & D.T. & $\mathrm{M}$ & D.T. & \\
\hline $\begin{array}{l}\text { Comprender la importancia del tra- } \\
\text { bajo coordinado en mi futuro profe- } \\
\text { sional como docente }\end{array}$ & 4.22 & .888 & 4.34 & .832 & 4.09 & 1.044 & .090 \\
\hline $\begin{array}{l}\text { Llegar a acuerdos ante opiniones } \\
\text { diferentes }\end{array}$ & 4.11 & .901 & 4.30 & .813 & 4.18 & .929 & .158 \\
\hline $\begin{array}{l}\text { Entender los conocimientos e ideas } \\
\text { de los compañeros y compañeras }\end{array}$ & 4.06 & .850 & 4.26 & .871 & 4.03 & .891 & .064 \\
\hline $\begin{array}{l}\text { Sentirme parte activa de mi propio } \\
\text { proceso de aprendizaje }\end{array}$ & 4.03 & .907 & 4.23 & .964 & 4.02 & .986 & .109 \\
\hline $\begin{array}{l}\text { Buscar información, investigar y } \\
\text { aprender de forma autónoma }\end{array}$ & 4.05 & .978 & 4.31 & .837 & 3.89 & 1.033 & .001 \\
\hline $\begin{array}{l}\text { Exponer y defender mis ideas y } \\
\text { conocimientos ante otras personas }\end{array}$ & 3.95 & .949 & 4.28 & .829 & 4.05 & .871 & .004 \\
\hline
\end{tabular}


Los alumnos de segundo priorizan las contribuciones del aprendizaje en grupo de manera similar, no obstante puede apreciarse una diferencia destacable y es que valoran como de mucha más importancia al trabajo coordinado en mi futuro profesional como docente.

Por su parte, los alumnos de tercero priorizan las contribuciones del aprendizaje en grupo de manera similar a los de primero, no obstante puede apreciarse una diferencia destacable y es que valoran como de mucha más importancia llegar a acuerdos ante opiniones diferentes. Por todo ello y debido a la diferencia entre los tres grupos, las estrategias de buscar información, investigar y aprender de forma autónoma y exponer y defender mis ideas y conocimientos ante otras personas se convierte así en las únicas diferencias estadísticamente significativa entre los tres grupos, con unas $\mathrm{p}=.001$ y $\mathrm{p}=.004$ respectivamente.

Los alumnos de primero se muestran desde de acuerdo hasta ni en acuerdo ni en desacuerdo con las siguientes dinámicas: la consulta de la documentación del docente (4.11), la búsqueda de información en otras fuentes (4.07), la toma consensuada de decisiones para aumentar la coherencia (4.04), la puesta en común del trabajo realizado (3.98), la reunión para la organización del trabajo (3.95), la realización de una evaluación y propuesta de mejora (3.73) y la participación equitativa (3.58) (Tabla 3).

Mientras tanto, los alumnos de segundo les dan una puntuación superior y valoran sobre el resto de estrategias la búsqueda de información adicional (4.38), seguida de la consulta de la documentación del docente y la toma consensuada de decisiones (ambas con un 4.29) y la puesta en común (4.21).

Así los alumnos de tercero, obtienen valores más similares a los de primero aunque inferiores, obteniendo las puntuaciones superiores en las estrategias la búsqueda de información adicional y puesta en común (4.05), seguida de toma consensuada de decisiones (4.03).

La evaluación para realizar propuestas de mejora, la toma consensuada de decisiones, la búsqueda de información en otras fuentes y la consulta de la documentación del docente son donde se encuentran diferencias estadísticamente significativas entre los tres grupos, tal y como evidencia la distancia entre sus medias, resultando de su comparación con la prueba ANOVA menor a 05.

Tabla 3

Comparación del funcionamiento interno de los grupos en alumnos de primero, segundo y tercero

\begin{tabular}{|c|c|c|c|c|c|c|c|}
\hline \multirow[t]{2}{*}{$\mathrm{Al}$ hacer un trabajo de grupo: } & \multicolumn{2}{|c|}{ Primero, $n=250$} & \multicolumn{2}{|c|}{ Segundo, $n=121$} & \multicolumn{2}{|c|}{$\begin{array}{c}\text { Tercero, } \mathrm{n}= \\
152\end{array}$} & \multirow[t]{2}{*}{$\mathrm{p}$} \\
\hline & $\mathrm{M}$ & D.T. & $\mathrm{M}$ & D.T. & $\mathrm{M}$ & D.T. & \\
\hline $\begin{array}{l}\text { Consultamos la documentación bási- } \\
\text { ca aportada por el profesor/a }\end{array}$ & 4.11 & .866 & 4.29 & .944 & 3.93 & 1.049 & .007 \\
\hline $\begin{array}{l}\text { Realizamos búsqueda de información } \\
\text { en diferentes fuentes (internet, biblio- } \\
\text { teca...) }\end{array}$ & 4.07 & .999 & 4.38 & .829 & 4.05 & 1.019 & .007 \\
\hline
\end{tabular}




\begin{tabular}{|c|c|c|c|c|c|c|c|}
\hline \multirow{2}{*}{$\mathrm{Al}$ hacer un trabajo de grupo: } & \multicolumn{2}{|c|}{ Primero, $n=250$} & \multicolumn{2}{|c|}{ Segundo, $\mathrm{n}=121$} & \multicolumn{2}{|c|}{$\begin{array}{c}\text { Tercero, } \mathrm{n}= \\
152\end{array}$} & \multirow{2}{*}{$\mathrm{p}$} \\
\hline & $\mathrm{M}$ & D.T. & $\mathrm{M}$ & D.T. & $\mathrm{M}$ & D.T. & \\
\hline $\begin{array}{l}\text { Hacemos "puestas en común" para } \\
\text { que todo el grupo conozca lo que los } \\
\text { demás están haciendo y tengamos } \\
\text { buena idea de la marcha de la activi- } \\
\text { dad }\end{array}$ & 3.98 & .994 & 4.21 & .865 & 4.05 & 1.022 & .120 \\
\hline $\begin{array}{l}\text { Nos reunimos al inicio para planifi- } \\
\text { car los diferentes pasos que tenemos } \\
\text { que realizar }\end{array}$ & 3.95 & 1.009 & 4.01 & 1.122 & 3.73 & 1.098 & .058 \\
\hline $\begin{array}{l}\text { Lo evaluamos y hacemos propuestas } \\
\text { de mejora }\end{array}$ & 3.73 & 1.108 & 4.04 & 1.012 & 3.20 & 1.266 & .000 \\
\hline $\begin{array}{l}\text { Participamos equitativamente todos } \\
\text { los componentes del grupo }\end{array}$ & 3.58 & 1.227 & 3.71 & 1.255 & 3.40 & 1.252 & .114 \\
\hline
\end{tabular}

Y en relación al segundo objetivo, los datos de las Tablas 4, 5 y 6 aportan que sí afecta directamente al concepto que los alumnos tienen del trabajo en grupo y a lo que aprenden de él y con él. Aunque los alumnos ya estén satisfechos o no satisfechos con el trabajo en grupo, con su aporte y con la contribución de sus compañeros, ordenan las características de su concepto del trabajo en grupo de manera similar a la que se presentó en el objetivo 1, encontrándose diferencias estadísticamente significativas en casi todos los enunciados debido a la distancia entre las puntuaciones que otorgan los alumnos satisfechos, que se aproximan a las ya descritas, y las que presentan los alumnos no satisfechos.

Tabla 4

Comparación de la concepción del trabajo en grupo según su satisfacción con el trabajo en grupo realizado

\begin{tabular}{|c|c|c|c|c|c|c|}
\hline \multirow{2}{*}{ El trabajo en grupo es: } & \multicolumn{2}{|c|}{ Sí satisfechos, $\mathrm{n}=471$} & \multicolumn{3}{|c|}{ No satisfechos, $n=52$} & \multirow{2}{*}{$\begin{array}{c}\mathrm{p} \\
(\mathrm{A}, \mathrm{K})\end{array}$} \\
\hline & $\mathrm{M}$ & D.T. & $\mathrm{M}$ & D.T. & K-S & \\
\hline $\begin{array}{l}\text { Una oportunidad para conocer mejor a } \\
\text { mis compañeros/as }\end{array}$ & 4.52 & .705 & 3.98 & .939 & .002 & $.000(\mathrm{U})$ \\
\hline $\begin{array}{l}\text { Un buen método para desarrollar mis } \\
\text { competencias sociales: argumentación, } \\
\text { diálogo capacidad de escucha, debate, } \\
\text { respeto a opiniones discrepantes... }\end{array}$ & 4.42 & .725 & 3.73 & 1.069 & .007 & $.000(\mathrm{U})$ \\
\hline $\begin{array}{l}\text { Una manera de compartir el volumen } \\
\text { de trabajo total }\end{array}$ & 4.18 & .943 & 3.10 & 1.302 & .071 & $.000(t)$ \\
\hline $\begin{array}{l}\text { Una forma de comprender mejor los } \\
\text { conocimientos }\end{array}$ & 4.08 & .896 & 3.17 & 1.133 & .079 & $.015(t)$ \\
\hline $\begin{array}{l}\text { Una manera de facilitar la preparación } \\
\text { de los exámenes }\end{array}$ & 3.61 & 1.115 & 2.50 & 1.365 & .020 & $.000(\mathrm{U})$ \\
\hline
\end{tabular}

Nota: $\mathrm{K}-\mathrm{S}=$ sig. bilateral de prueba de Kolmogorov-Smirnov; $\mathrm{p}=$ grado de significación; $\mathrm{A}=$ prueba ANOVA para muestras independientes; $\mathrm{K}=$ Kruskal-Wallis para muestras independientes. 
Tabla 5

Comparación de la concepción del trabajo en grupo según su satisfacción con el aporte personal

\begin{tabular}{|c|c|c|c|c|c|c|}
\hline \multirow[t]{2}{*}{ El trabajo en grupo es: } & \multicolumn{2}{|c|}{$\begin{array}{l}\text { Sí satisfechos, } \mathrm{n}= \\
501\end{array}$} & \multicolumn{3}{|c|}{ No satisfechos, $\mathrm{n}=22$} & \multirow[t]{2}{*}{$p(A, K)$} \\
\hline & $\mathrm{M}$ & D.T. & M & D.T. & K-S & \\
\hline $\begin{array}{l}\text { Una oportunidad para conocer mejor } \\
\text { a mis compañeros/as }\end{array}$ & 4.52 & .705 & 3.98 & .939 & .029 & $\begin{array}{l}.580 \\
(\mathrm{U})\end{array}$ \\
\hline $\begin{array}{l}\text { Un buen método para desarrollar } \\
\text { mis competencias sociales: argu- } \\
\text { mentación, diálogo, capacidad de } \\
\text { escucha, debate, respeto a opiniones } \\
\text { discrepantes... }\end{array}$ & 4.42 & .752 & 3.73 & 1.069 & .112 & $.018(\mathrm{t})$ \\
\hline $\begin{array}{l}\text { Una manera de compartir el volumen } \\
\text { de trabajo total }\end{array}$ & 4.18 & .943 & 3.10 & 1.302 & .057 & $.746(t)$ \\
\hline $\begin{array}{l}\text { Una forma de comprender mejor los } \\
\text { conocimientos }\end{array}$ & 4.08 & .896 & 3.17 & 1.133 & .248 & $.001(t)$ \\
\hline $\begin{array}{l}\text { Una manera de facilitar la prepara- } \\
\text { ción de los exámenes }\end{array}$ & 3.01 & 1.115 & 2.50 & 1.365 & .461 & $.483(t)$ \\
\hline
\end{tabular}

Nota: $\mathrm{K}-\mathrm{S}=$ sig. bilateral de prueba de Kolmogorov-Smirnov; $\mathrm{p}=$ grado de significación; A= prueba ANOVA para muestras independientes; $\mathrm{K}=\mathrm{Kruskal}-$ Wallis para muestras independientes.

Tabla 6

Comparación de la concepción del trabajo en grupo según su satisfacción con la contribución de los compañeros

\begin{tabular}{|c|c|c|c|c|c|c|}
\hline \multirow{2}{*}{ El trabajo en grupo es: } & \multicolumn{2}{|c|}{ Sí satisfechos, $n=443$} & \multicolumn{3}{|c|}{ No satisfechos, $n=80$} & \multirow{2}{*}{$\begin{array}{c}\mathrm{p} \\
(\mathrm{A}, \mathrm{K})\end{array}$} \\
\hline & $\mathrm{M}$ & D.T. & $\mathrm{M}$ & D.T. & K-S & \\
\hline $\begin{array}{l}\text { Una oportunidad para conocer mejor a } \\
\text { mis compañeros/as }\end{array}$ & 4.51 & .713 & 4.17 & .868 & .000 & $.000(\mathrm{U})$ \\
\hline $\begin{array}{l}\text { Un buen método para desarrollar mis } \\
\text { competencias sociales: argumentación, } \\
\text { diálogo, capacidad de escucha, debate, } \\
\text { respeto a opiniones discrepantes... }\end{array}$ & 4.43 & .740 & 3.95 & .940 & .010 & $.000(\mathrm{U})$ \\
\hline $\begin{array}{l}\text { Una manera de compartir el volumen de } \\
\text { trabajo total }\end{array}$ & 4.22 & .912 & 3.28 & 1.292 & .000 & $\begin{array}{l}.000 \\
(\mathrm{U})\end{array}$ \\
\hline $\begin{array}{l}\text { Una forma de comprender mejor los } \\
\text { conocimientos }\end{array}$ & 4.09 & .899 & 3.43 & 1.088 & .006 & $\begin{array}{l}.000 \\
(\mathrm{U})\end{array}$ \\
\hline $\begin{array}{l}\text { Una manera de facilitar la preparación } \\
\text { de los exámenes }\end{array}$ & 3.60 & 1.112 & 2.90 & 1.411 & .010 & $\begin{array}{l}.000 \\
(\mathrm{U})\end{array}$ \\
\hline
\end{tabular}

Nota: $\mathrm{K}-\mathrm{S}=$ sig. bilateral de prueba de Kolmogorov-Smirnov; $\mathrm{p}=$ grado de significación; $\mathrm{A}=$ prueba ANOVA para muestras independientes; $K=$ Kruskal-Wallis para muestras independientes. 
Los alumnos no satisfechos con el trabajo en grupo, con su aporte personal o con la contribución de sus compañeros, están tan sólo levemente de acuerdo con que el trabajo en grupo les permite conocer mejor a sus compañeros y, en el caso de los alumnos no satisfechos con su aporte personal, con que es una forma de distribuir equitativamente las tareas. Además, los alumnos no satisfechos se muestran en desacuerdo con que el trabajo en grupo les permita prepararse para los exámenes.

Como puede verse en las tablas 7, 8 y 9, mientras que entre los alumnos satisfechos con el trabajo en grupo, con su aporte o con la contribución de sus compañeros, puede haber pequeñas diferencias en la ordenación de las dinámicas que se usan. Sin embargo, entre los alumnos no satisfechos, las puntuaciones vuelven a descender. El único punto en el que parecen estar todos de acuerdo es en la baja importancia relativa que le dan a las estrategias de hacer propuestas de mejora o participar de manera equitativa.

Por último, existen diferencias significativas entre los alumnos satisfechos y los no satisfechos con el funcionamiento del trabajo en grupo en todos los enunciados (Tabla 7).

Tabla 7

Comparación del funcionamiento interno de los grupos según su satisfacción con el trabajo en grupo realizado

\begin{tabular}{|c|c|c|c|c|c|c|}
\hline \multirow{2}{*}{ Al hacer un trabajo de grupo: } & \multicolumn{2}{|c|}{ Sí satisfechos, $n=471$} & \multicolumn{3}{|c|}{ No satisfechos, $n=52$} & \multirow{2}{*}{$\begin{array}{c}\mathrm{p} \\
(\mathrm{A}, \mathrm{K})\end{array}$} \\
\hline & $\mathrm{M}$ & D.T. & M & D.T. & K-S & \\
\hline $\begin{array}{l}\text { Tomamos decisiones, de forma consen- } \\
\text { suada, para garantizar la coherencia } \\
\text { global del trabajo de grupo }\end{array}$ & 4.21 & .870 & 3.08 & 1.152 & .008 & $.000(\mathrm{U})$ \\
\hline $\begin{array}{l}\text { Realizamos búsqueda de información } \\
\text { en diferentes fuentes (internet, biblio- } \\
\text { teca,...) }\end{array}$ & 4.19 & .957 & 3.65 & 1.027 & .021 & $.000(\mathrm{U})$ \\
\hline $\begin{array}{l}\text { Hacemos "puestas en común" para que } \\
\text { todo el grupo conozca lo que los demás } \\
\text { están haciendo y tengamos buena idea } \\
\text { de la marcha de la actividad }\end{array}$ & 4.16 & .889 & 3.10 & 1.192 & .070 & $.002(t)$ \\
\hline $\begin{array}{l}\text { Consultamos la documentación básica } \\
\text { aportada por el profesor/a }\end{array}$ & 4.15 & .909 & 3.63 & 1.155 & .005 & $.001(\mathrm{U})$ \\
\hline $\begin{array}{l}\text { Nos reunimos al inicio para planificar } \\
\text { los diferentes pasos que tenemos que } \\
\text { realizar }\end{array}$ & 3.97 & 1.012 & 3.27 & 1.315 & .044 & $.000(\mathrm{U})$ \\
\hline $\begin{array}{l}\text { Participamos equitativamente todos los } \\
\text { componentes del grupo }\end{array}$ & 3.75 & 1.108 & 1.87 & 1.121 & .000 & $.000(\mathrm{U})$ \\
\hline $\begin{array}{l}\text { Lo evaluamos y hacemos propuestas de } \\
\text { mejora }\end{array}$ & 3.77 & 1.100 & 2.56 & 1.290 & .083 & $.010(\mathrm{t})$ \\
\hline
\end{tabular}

Nota: $\mathrm{K}-\mathrm{S}=$ sig. bilateral de prueba de Kolmogorov-Smirnov; $\mathrm{p}=$ grado de significación; A= prueba ANOVA para muestras independientes; $\mathrm{K}=\mathrm{Kruskal}-$ Wallis para muestras independientes. 
Tabla 8

Comparación del funcionamiento interno de los grupos según su satisfacción con su aporte personal

\begin{tabular}{|c|c|c|c|c|c|c|}
\hline \multirow{2}{*}{ Al hacer un trabajo de grupo: } & \multicolumn{2}{|c|}{ Sí satisfechos, $\mathrm{n}=501$} & \multicolumn{3}{|c|}{ No satisfechos, $n=22$} & \multirow[b]{2}{*}{$\mathrm{p}(\mathrm{A}, \mathrm{K})$} \\
\hline & M & D.T. & M & D.T. & K-S & \\
\hline $\begin{array}{l}\text { Tomamos decisiones, de forma consen- } \\
\text { suada, para garantizar la coherencia } \\
\text { global del trabajo de grupo }\end{array}$ & 4.13 & .920 & 3.50 & 1.336 & .088 & $.009(\mathrm{t})$ \\
\hline $\begin{array}{l}\text { Realizamos búsqueda de información } \\
\text { en diferentes fuentes (internet, biblio- } \\
\text { teca,...) }\end{array}$ & 4.15 & .966 & 3.77 & 1.152 & .102 & $.501(\mathrm{t})$ \\
\hline $\begin{array}{l}\text { Hacemos "puestas en común" para que } \\
\text { todo el grupo conozca lo que los demás } \\
\text { están haciendo y tengamos buena idea } \\
\text { de la marcha de la actividad }\end{array}$ & 4.13 & .935 & 3.41 & 1.297 & .296 & $.018(\mathrm{t})$ \\
\hline $\begin{array}{l}\text { Consultamos la documentación básica } \\
\text { aportada por el profesor/a }\end{array}$ & 4.08 & .953 & 3.46 & 1.299 & .060 & $.004(\mathrm{t})$ \\
\hline $\begin{array}{l}\text { Nos reunimos al inicio para planificar } \\
\text { los diferentes pasos que tenemos que } \\
\text { realizar }\end{array}$ & 3.92 & 1.055 & 3.45 & 1.224 & .243 & $.357(\mathrm{t})$ \\
\hline $\begin{array}{l}\text { Participamos equitativamente todos los } \\
\text { componentes del grupo }\end{array}$ & 3.60 & 1.225 & 2.64 & 1.329 & .598 & $.525(\mathrm{t})$ \\
\hline $\begin{array}{l}\text { Lo evaluamos y hacemos propuestas de } \\
\text { mejora }\end{array}$ & 3.68 & 1.157 & 2.86 & 1.356 & .296 & $.152(t)$ \\
\hline
\end{tabular}

Nota: $\mathrm{K}-\mathrm{S}=$ sig. bilateral de prueba de Kolmogorov-Smirnov; $\mathrm{p}=$ grado de significación; A= prueba ANOVA para muestras independientes; $\mathrm{K}=$ Kruskal-Wallis para muestras independientes.

No obstante, los alumnos que menos atención prestan a la documentación del docente son aquellos que dicen no estar satisfechos con su aporte personal (3.46), ya que estos consideran mucho más importante la búsqueda de información en diferentes fuentes, seguido de tomar decisiones consensuadas y de reunirnos para planificar.

Los alumnos no satisfechos con el trabajo de sus compañeros (Tabla 9) colocan como estrategias más destacadas el consultar la información del docente y buscar información adicional en otras fuentes, para después hacer puestas en común para informar de los progresos, después tomar algunas decisiones consensuadas y finalmente reunirse. Es destacable que estos alumnos son los que menos de acuerdo están con que dentro del grupo el reparto de la tarea sea equitativo. 
Tabla 9

Comparación del funcionamiento interno de los grupos según su satisfacción con la contribución de los compañeros

\begin{tabular}{|c|c|c|c|c|c|c|}
\hline \multirow{2}{*}{ Al hacer un trabajo de grupo: } & \multicolumn{2}{|c|}{ Sí satisfechos, $\mathrm{n}=443$} & \multicolumn{3}{|c|}{ No satisfechos, $\mathrm{n}=80$} & \multirow{2}{*}{$\begin{array}{c}\mathrm{p} \\
(\mathrm{A}, \mathrm{K})\end{array}$} \\
\hline & $\mathrm{M}$ & D.T. & $\mathrm{M}$ & D.T. & K-S & \\
\hline $\begin{array}{l}\text { Tomamos decisiones, de forma consen- } \\
\text { suada, para garantizar la coherencia } \\
\text { global del trabajo de grupo }\end{array}$ & 4.22 & .851 & 3.41 & 1.229 & .000 & $.000(\mathrm{U})$ \\
\hline $\begin{array}{l}\text { Realizamos búsqueda de información } \\
\text { en diferentes fuentes (internet, biblio- } \\
\text { teca,...) }\end{array}$ & 4.20 & .941 & 3.76 & 1.082 & .001 & $.000(\mathrm{U})$ \\
\hline $\begin{array}{l}\text { Hacemos "puestas en común" para que } \\
\text { todo el grupo conozca lo que los demás } \\
\text { están haciendo y tengamos buena idea } \\
\text { de la marcha de la actividad }\end{array}$ & 4.16 & .886 & 3.49 & 1.232 & .000 & $.000(\mathrm{U})$ \\
\hline $\begin{array}{l}\text { Consultamos la documentación básica } \\
\text { aportada por el profesor/a }\end{array}$ & 4.16 & .915 & 3.79 & 1.064 & .000 & $.003(\mathrm{U})$ \\
\hline $\begin{array}{l}\text { Nos reunimos al inicio para planificar } \\
\text { los diferentes pasos que tenemos que } \\
\text { realizar }\end{array}$ & 4.00 & .984 & 3.32 & 1.300 & .000 & $.000(\mathrm{U})$ \\
\hline $\begin{array}{l}\text { Participamos equitativamente todos los } \\
\text { componentes del grupo }\end{array}$ & 3.83 & 1.046 & 2.05 & 1.168 & .000 & $.000(\mathrm{U})$ \\
\hline $\begin{array}{l}\text { Lo evaluamos y hacemos propuestas de } \\
\text { mejora }\end{array}$ & 3.78 & 1.104 & 2.89 & 1.273 & .035 & $.000(\mathrm{U})$ \\
\hline
\end{tabular}

Nota: $\mathrm{K}-\mathrm{S}=$ sig. bilateral de prueba de Kolmogorov-Smirnov; $\mathrm{p}=$ grado de significación; A= prueba ANOVA para muestras independientes; $\mathrm{K}=$ Kruskal-Wallis para muestras independientes.

\section{Discusión y Conclusiones}

Los resultados arrojan que los alumnos de Educación Primaria se forman rápidamente una idea acerca del concepto del trabajo en grupo y que ésta se mantiene a lo largo del tiempo. Los estudiantes no aprecian que el uso de esta metodología esté asociado directamente al desarrollo de los conocimientos teóricos necesarios para adquirir la titulación, sino que lo consideran una oportunidad para desarrollar aspectos personales de relación entre compañeros a la par que incrementan sus habilidades y competencias sociales en un ambiente controlado y más próximo al laboral.

Los alumnos así lo reconocen cuando admiten que las principales utilidades que encuentran a esta metodología son que les hace conscientes de la importancia del trabajo coordinado para su futura profesión y les ayuda a establecer acuerdos de trabajo con otras personas que difieren con sus puntos de vista, además de que durante el proceso aprenden a expresar y defender sus propias ideas al respecto de temas profesionales. 
Investigaciones de otros autores (Escarbajal, 2010; Merino et al., 2012) también verifican que tanto profesores como estudiantes y egresados perciben que las metodologías activas, en las que la evaluación es sumativa, ayudan a los alumnos a adquirir competencias profesionales.

Es por medio de este proceso que los alumnos toman consciencia del valor añadido que el trabajo en grupo posee para la consecución de sus objetivos, a la vez que aprenden a valorar las contribuciones que hacen otras personas a su trabajo y encontrar en estas otras personas una fuente de apoyo y enriquecimiento. Esto evidencia la percepción que tienen los alumnos de los miembros de su propio grupo como individuos comprometidos con su trabajo y con el éxito del grupo que se interesan por hacer bien su labor (Gavilán \& Alario, 2010).

Como bien indicaban expertos en autorregulación como Pintrich (2004) y Gaeta y Herrero (2009), las reacciones afectivas, juicios y opiniones emitidos una vez acabado un trabajo, juegan un papel esencial en la actitud con la que los alumnos enfrentan futuras tareas y con la confianza en sus propias aptitudes que manejan en esas nuevas circunstancias.

Con el fin de especificar mejor a qué era debida la satisfacción o insatisfacción, se distinguen tres situaciones distintas en las que el alumno podía sentirse satisfecho o insatisfecho: con el trabajo del grupo, con la contribución de los compañeros al desarrollo del trabajo en grupo y con el aporte personal al mismo.

Al comparar los alumnos satisfechos con los insatisfechos, se encuentra que, mientras los alumnos satisfechos seguían obteniendo valores próximos a los ya descritos, cuando se hace referencia a la progresión del alumnado en la titulación, los alumnos insatisfechos, independientemente del aspecto concreto que se tratara, valoraban sus cuestionarios con puntuaciones inferiores o muy inferiores. De esto se deduce que la satisfacción es efectivamente un factor que influye tanto en la concepción del trabajo en grupo y el uso de dinámicas de trabajo en grupo, como en la percepción que tienen los alumnos de sus propios compañeros.

Con respecto al trabajo en grupo, los alumnos insatisfechos desarrollaban ideas similares a los satisfechos, sin embargo la intensidad y convicción tras estas ideas era mucho menor, especialmente de los estudiantes insatisfechos con sus compañeros o con el trabajo en grupo. Es comprensible que tengan un peor concepto del trabajo en grupo una vez que se indaga en los beneficios que dicen que les reporta y en las dinámicas que emplean.

En este punto hay que hacer una distinción entre los alumnos no satisfechos con el trabajo en sí, y los alumnos no satisfechos con las personas involucradas en el trabajo. Los alumnos insatisfechos con el trabajo en grupo emplean menos las dinámicas en las que se participa y crea conocimiento compartido con otros alumnos y, además, ni creen ni dejan de creer que el trabajo en grupo les ayude a desarrollar competencias docentes.

\section{Referencias}

Abal, F. J. P., Lozzia, G. S., Aguerri, M.E., Galibert, M. S. \& Attorresi, H. F. (2008). Evidencias acerca de la validez de constructo de la escala voluntad de trabajo. Anuario de Investigaciones, 15, 283-289. Recuperado de https://www.redalyc.org /articulo. oa?id=369139944027 
Agencia Nacional de Evaluación de la Calidad y Acreditación [ANECA] (2004). Título de grado en magisterio. Volumen 1. Recuperado de http://www.aneca.es/var/media/150404/ libroblanco_jun05_magisterio1.pdf

Andrés, A., Abal, F. J. P., Lozzia, G. S., Gómez-Benito, J., Aguerri, M. E., Galibert, M. S. \& Attorresi, H. F. (2013). Adaptación del Cuestionario de voluntad de trabajo a una muestra de universitarios españoles. Anales de Psicología, 29(1), 171-177. doi: https:// doi.org/10.6018/analesps.29.1. 124531

Antúnez, S. (1999). El trabajo en equipo de los profesores y profesoras: Factor de calidad, necesidad y problema. El papel de los directivos escolares. Educar, 24, 89-110. doi: https://doi.org /10.5565/rev/educar.337

Colomer, M., Durán, H. \& Puigcerver, M. (2016). Una nueva manera de utilizar los itinerarios urbanos en la enseñanza de la Geología: trabajo cooperativo mediante computación en la nube. Enseñanza de las Ciencias de la Tierra, 24(2), 202-212. Recuperado de https://dialnet.unirioja.es/servlet/articulo?codigo=5779654

Conde, J., Martín, A. \& Torres, C. (2012). Adquisición de competencias docentes a través de estudio de casos. En E. Nieto, A. I. Callejas, \& Ó. Jerez (Eds.), Las competencias básicas. Competencias profesionales del docente (pp. 535-544). Ciudad Real: Universidad de Castilla-La Mancha. Servicio de Publicaciones.

Domingo, J. (2008). El aprendizaje cooperativo. Cuadernos de Trabajo Social, 21, 231-246. Recuperado de https://core.ac.uk/download/pdf/38812746.pdf

Escarbajal, A. (2010). Interculturalidad, mediación y trabajo cooperativo. Madrid: Narcea.

Gaeta, M. L. \& Herrero, M. L. (2009). Influencia de las estrategias volitivas en la autorregulación del aprendizaje. Estudios de Psicología, 30, 73-88. doi: 10.1174/021093909787536317.

Galán, J. I. \& Fuentes, P. (1999). Trabajo en grupo/trabajo agrupado: Realidades en el aula. Revista de Investigación Educativa, 17(2), 559-569. Recuperado de https://revistas. um.es/rie/article /view/122201/114841

García, M. M., González, I. \& Mérida, R. (2012). Validación del cuestionario de evaluación ACOES. Análisis del trabajo cooperativo en educación superior. Revista de Investigación Educativa, 30(1), 87-109. doi: https://doi.org/10.6018/rie.30.1.114091

Gavilán, P. \& Alario, R. (2010). Aprendizaje cooperativo. Una metodología con futuro. Principios y aplicaciones. Madrid: CCS.

Gómez, M. P. \& Gómez, A. H. (2016). Experiencia docente acerca del uso didáctico del aprendizaje cooperativo y el trabajo de campo en el estudio del fenómeno de influencia social. Revista Electrónica Interuniversitaria de Formación del Profesorado, 19(2), 331-346. doi: https://doi.org/10.6018/reifop.19.2.206921

Hernández, E. (2006). Metodología de la investigación: Cómo escribir una tesis. Recuperado de http://cmapspublic.ihmc.us/rid=1HWFWBKV2-27SKQ71-14CF/Como\%20escribir.pdf

Hernández, R., Fernández, C. \& Baptista, M. P. (2010). Metodología de la investigación (5 $5^{\underline{a}}$ ed.). México D.F.: McGraw-Hill.

Iglesias, J., González, L. \& Fernández-Río, J. (2017). Aprendizaje cooperativo. Madrid: Ediciones Pirámide.

Instituto Nacional para el Empleo (INEM). (1995). Metodología para la ordenación de la formación profesional ocupacional. Madrid: Subdirección General de Gestión de Formación Ocupacional. 
Izquierdo, T., Escarbajal, A. \& Latorre, P.A. (2016). Motivaciones que condicionan la formación y previenen la exclusión social de los futuros educadores. Revista de Investigación Educativa, 34(2), 285-298. doi: https://doi.org/10.6018/rie.34.2.238381

Johnson, D. W., Johnson, R.T. \& Holubec E.J. (1999). El aprendizaje cooperativo en el aula. Buenos Aires: Paidós.

Krichesky, G. J. \& Murillo, F. J. (2018). La colaboración docente como factor de aprendizaje y promotor de mejora: Un estudio de casos. Educacion XX1, 21(1), 135-156. doi: https://doi.org/10.5944/educxx1.20181

Laal, M. \& Laal, M. (2012). Collaborative learning: What is it? Procedia. Social and Behavioral Sciences, 31, 491-495. doi: https://doi.org/10.1016/j.sbspro.2011.12.092

Merino, C., Cortón, M. O. \& Cortón M. T. (2012). Percepción del desarrollo de competencias profesionales en la formación inicial del profesorado en las universidades de Castilla y León. En E. Nieto, A. I. Callejas \& Ó. Jerez (Eds.), Las competencias básicas. Competencias profesionales del docente (pp. 169-178). Ciudad Real: Universidad de Castilla-La Mancha. Servicio de Publicaciones.

Murillo, J., Krichesky, G., Castro, A. \& Reyes, C. (2010). Liderazgo para la inclusión escolar y la justicia social. Aportaciones de la investigación. Revista Latinoamericana de Inclusión Educativa, 4(1), 169-186. Recuperado de http://www.rinace.net/rlei/ numeros/vol4-num1/art8.pdf

Panitz, T. (1999). Collaborative versus cooperative learning: A comparison of the two concepts which will help us understand the underlying nature of interactive learning, 1-13. Recuperado de https://eric.ed.gov/?id=ED448443.

Perrenoud, P. (2004). Diez nuevas competencias para enseñar. Barcelona: Graó.

Pintrich, P. R. (2004). A conceptual framework for assessing motivation and selfregulated learning in college students. Educational Psychology Review, 16(4), 385-407. doi: http://dx.doi.org/10.1007/s10648-004-0006-x

Rondón, E., Salas, M., González, V., Martínez, P. \& González, A. (2018). El aprendizaje cooperativo en la enseñanza de la matematica. Impacto Científico, 12(2), 189-201.

Serrano, J. M. (1996). El aprendizaje cooperativo. En J.L. Beltrán \& C. Genovard (Eds.), Psicología de la Instrucción I. Variables y procesos básicos (pp. 217-244). Madrid: Síntesis.

Stortoni, M. (2016). El rol docente en los grupos de ingresantes universitarios. Escritos en la Facultad, 37, 37-39. Recuperado de https://fido.palermo.edu/servicios_dyc/ publicacionesdc/ archivos/624_libro.pdf

Vázquez, A. (2013). Interdependencia entre el liderazgo transformacional, cultura organizacional y cambio educativo: una reflexión. Revista Iberoamericana sobre Calidad, Eficacia y Cambio en Educación, 11(1), 74-91. Recuperado de http://www.redalyc.org/ pdf/551/55125665006.pdf

Fecha de recepción: 22 de marzo de 2019.

Fecha de revisión: 25 de marzo de 2019.

Fecha de aceptación: 8 de abril de 2019. 
